\section{New Technique for Examining Recording Media and other Magnetic Materials}

Magnetic Force Microscopy (MFM) is a recent application of atomic force microscopy that allows imaging of magnetic fields near surfaces. Applications include examination of magnetic media (audio and video tapes and disks) as well as other magnetic materials. MFM provides resolution far superior to optical methods and requires little or no sample preparation; however, its usefulness has been limited by the inability to distinguish magnetic effects from topographic features. Hence, images have generally comprised a combination of both influences.

To solve this problem, a new technique called LiftMode ${ }^{T M}$ has been introduced by Digital Instruments for its NanoScope ${ }^{\text {TM }}$ line of AFMs. LiftMode is a scanning technique that provides separate images for topography and magnetic force and virtually eliminates cross-contamination of the images. To achieve these separate images, the sample is first scanned for topography using Digital's unique TappingMode ${ }^{T M}$ technique. The NanoScope then performs a second scan to collect the magnetic force image, using LiftMode to adjust for the topography and maintain a constant distance between the scanning tip and the sample. Digital Instruments has applied for patents for both LiftMode and TappingMode.

The images below show both the topographic features (left) and the magnetic force (center) for an experimental magneto-optical disk. The power of the laser used to write these bits was purposely varied, creating magnetic bits of different sizes. Notice the crisp resolution of the magnetic bits the smallest bit shown is less than $90 \mathrm{~nm}$ across. Magnetization is oriented perpendicular to the sample plane, opposite in direction to the background magnetization.

The LiftMode technique also excels at imaging magnetically "soft" materials, such as thin Permalloy films and garnet films. Soft materials pose a particular challenge for MFM because the domains are easily influenced by stray fields emanating from the magnetized tip. The image below (right) shows magnetic force images of three $90 \mathrm{~nm}$ thick Permalloy films on glass. These films are topographically identical but are magnetically quite different, as is apparent from the image.

The MFM LifMode technique provides the highest resolution images available for a variety of magnetic media, including magneto-optical disks, hard disks, recording tape and video tape, as well as other magnetic materials. All images were acquired using NanoProbe ${ }^{T M}$ magnetically coated tips which are specifically designed for magnetic applications.

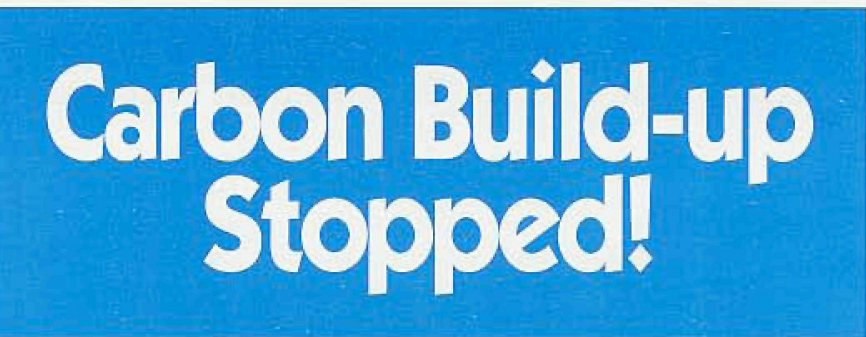

\section{Applications Lab Secret Revealed:}
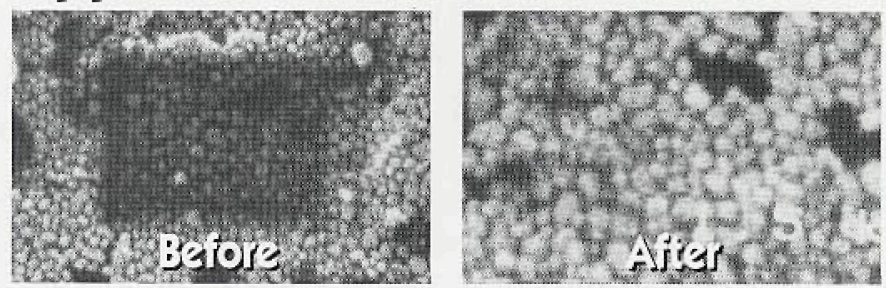

SEM manufacturers won't admit it, but most SEMs are subject to contamination build-up-even dry pumped systems. To stop hydrocarbon condensation, major applications labs and SEM users rely on the XEI Scientific SEM-CLEAN"' system.

Result: Outstanding pictures at low $\mathrm{kV}$ and high resolution and no oil on EDS X-ray detector windows. The Nitrogen purge of the inexpensive SEM-CLEAN system cleans your electron microscope while you're away.

\section{SEM-CLEAN"m Stops the Oil

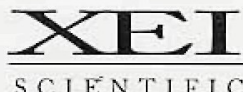

3124 Wessex Way, Redwood City, CA 94061 - 415-369-0133 - Fax 415-363-1659

How many consultants does it take to change a light bulb?

One. That will be a hundred dollars, please.
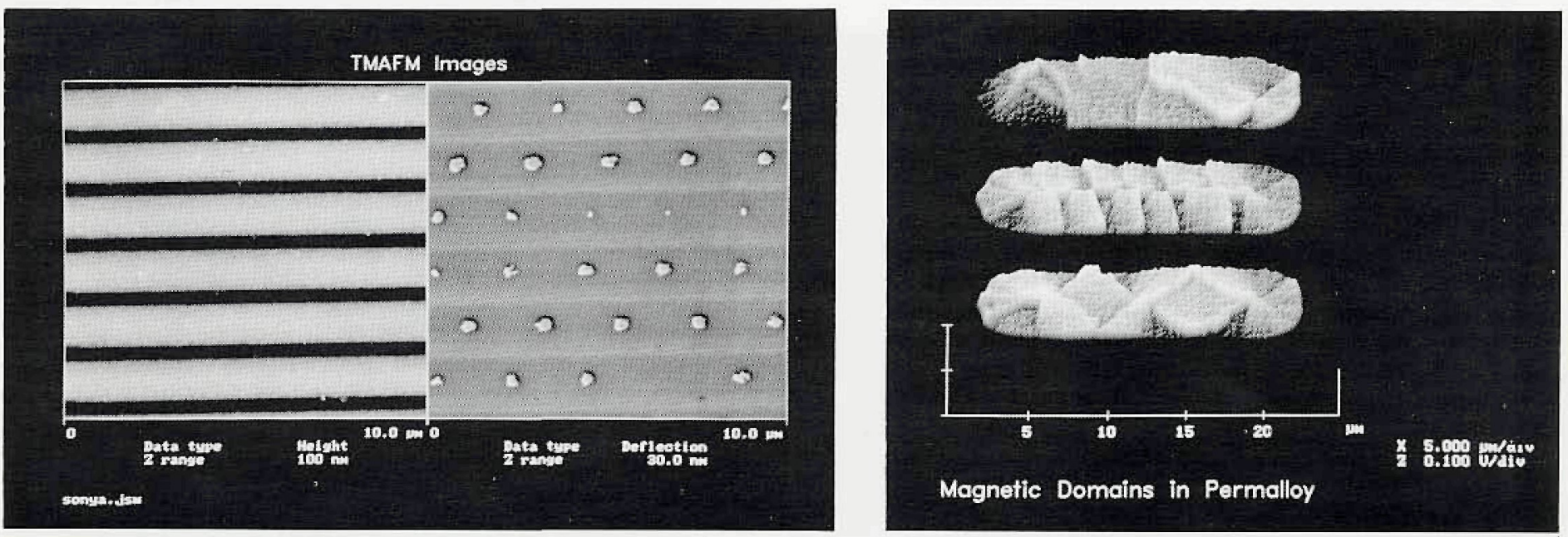